\title{
10^(10^6) Worlds and Beyond: Efficient Representation and Processing of Incomplete Information
}

\author{
Lyublena Antova \\ Cornell University \\ lantova@cs.cornell.edu
}

\author{
Christoph Koch \\ Cornell University \\ koch@cs.cornell.edu
}

\author{
Dan Olteanu \\ Oxford University \\ dan.olteanu@comlab.ox.ac.uk
}

\begin{abstract}
We present a decomposition-based approach to managing incomplete information. We introduce world-set decompositions (WSDs), a space-efficient and complete representation system for finite sets of worlds. We study the problem of efficiently evaluating relational algebra queries on world-sets represented by WSDs. We also evaluate our technique experimentally in a large census data scenario and show that it is both scalable and efficient.
\end{abstract}

\section{Introduction}

Incomplete information is commonplace in real-world databases. Classical examples can be found in data integration and wrapping applications, linguistic collections, or whenever information is manually entered and is therefore prone to inaccuracy or partiality.

There has been little research so far into expressive yet scalable systems for representing incomplete information. Current techniques can be classified into two groups. The first group includes representation systems such as $v$-tables [15] and or-set relations [16] which are not strong enough to represent the results of relational algebra queries within the same formalism. In v-tables the tuples can contain both constants and variables, and each combination of possible values for the variables yields a possible world. Relations with or-sets can be viewed as v-tables, where each variable occurs only at a single position in the table and can only take values from a fixed finite set, the or-set of the field occupied by the variable. The so-called $c$-tables [15] belong to the second group of formalisms. They extend v-tables with conditions specified by logical formulas over the variables, thus constraining the possible values. Although c-tables are a strong representation system, they have not found application in practice. The main reason for this is probably that managing c-tables directly is rather inefficient. Even very basic problems such as deciding whether a tuple is in at least one world represented by the c-table are intractable [3].
As a motivation, consider two manually completed forms that may originate from a census and which allow for more than one interpretation (Figure 1). For simplicity we assume that social security numbers consist of only three digits. For instance, Smith's social security number can be read either as " 185 " or as " 785 ". We can represent the available information using a relation with or-sets:

\begin{tabular}{c|ccc} 
(TID) & $\mathrm{S}$ & $\mathrm{N}$ & $\mathrm{M}$ \\
\hline$t_{1}$ & $\{185,785\}$ & Smith & $\{1,2\}$ \\
$t_{2}$ & $\{185,186\}$ & Brown & $\{1,2,3,4\}$
\end{tabular}

It is easy to see that this or-set relation represents $2 \cdot 2$. $2 \cdot 4=32$ possible worlds.

Given such an incompletely specified database, it must of course be possible to access and process the data. Two data management tasks shall be pointed out as particularly important, the evaluation of queries on the data and data cleaning [17, 13, 18], by which certain worlds can be shown to be impossible and can be excluded. The results of both types of operation turn out not to be representable by orset relations in general. Consider for example the integrity constraint that all social security numbers be unique. For our example database, this constraint excludes 8 of the 32 worlds, namely those in which both tuples have the value 185 as social security number. It is impossible to represent the remaining 24 worlds using or-set relations. This is an example of a constraint that can be used for data cleaning; similar problems are observed with queries, e.g., the query asking for pairs of persons with differing social security numbers.

What we could do is store each world explicitly using a table called a world-set relation of a given set of worlds. Each tuple in this table represents one world and is the concatenation of all tuples in that world (see Figure 2).

The most striking problem of world-set relations is their size. If we conduct a survey of 50 questions on a population of 200 million and we assume that one in $10^{4}$ answers can be read in just two different ways, we get $2^{10^{6}}$ worlds. Each such world is a substantial table of 50 columns and $2 \cdot 10^{8}$ rows. We cannot store all these worlds explicitly in a world-set relation (which would have $10^{10}$ columns and 


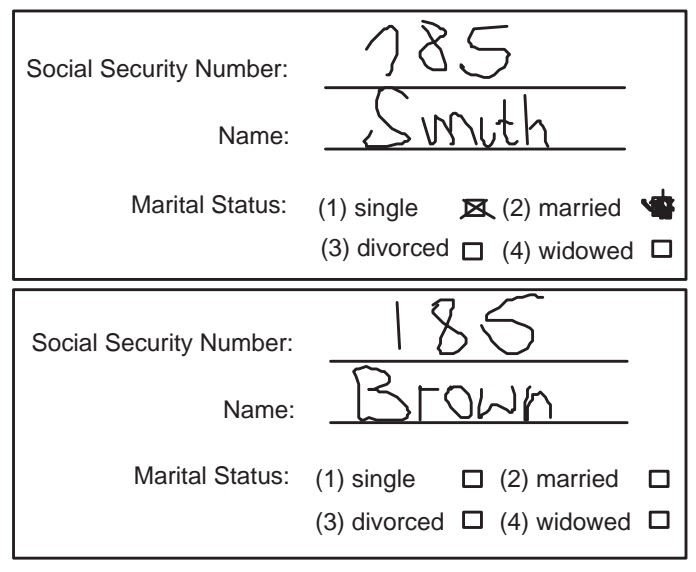

Figure 1. Two completed survey forms.

\begin{tabular}{cccccc}
$t_{1} . \mathrm{S}$ & $t_{1} . \mathrm{N}$ & $t_{1} \cdot \mathrm{M}$ & $t_{2} . \mathrm{S}$ & $t_{2} \cdot \mathrm{N}$ & $t_{2} . \mathrm{M}$ \\
\hline 185 & Smith & 1 & 186 & Brown & 1 \\
185 & Smith & 1 & 186 & Brown & 2 \\
185 & Smith & 1 & 186 & Brown & 3 \\
185 & Smith & 1 & 186 & Brown & 4 \\
185 & Smith & 2 & 186 & Brown & 1 \\
& & & & & \\
& & & & & \\
785 & Smith & 2 & 186 & Brown & 4 \\
\hline
\end{tabular}

Figure 2. World-set relation containing only worlds with unique social security numbers.

$2^{10^{6}}$ rows). Data cleaning will often eliminate only some of these worlds, so a DBMS should manage those that remain.

This article aims at dealing with this complexity and proposes the new notion of world-set decompositions (WSDs). These are decompositions of a world-set relation into several relations such that their product (using the product operation of relational algebra) is again the world-set relation.

Example 1.1 The world-set represented by our initial orset relation can also be represented by the product

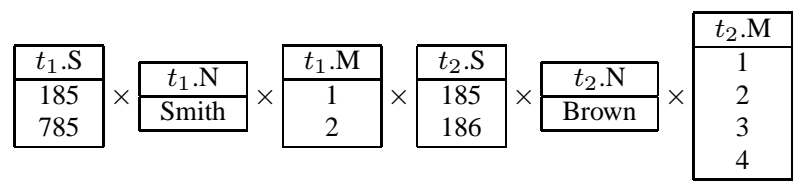

Example 1.2 In the same way we can represent the result of data cleaning with the uniqueness constraint for the social security numbers as the product of Figure 3 .

One can observe that the result of this product is exactly the world-set relation in Figure 2 The presented decomposition is based on the independence between sets of fields, subsequently called components. Only fields that depend on

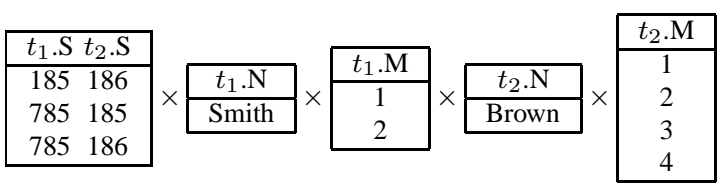

Figure 3. WSD of the relation in Figure 2.

each other, for example $t_{1} . S$ and $t_{2} . S$, belong to the same component. Since $\left\{t_{1} . S, t_{2} . S\right\}$ and $\left\{t_{1} . M\right\}$ are independent, they are put into different components.

Often, one can quantify the certainty of a combination of possible values using probabilities. For example, an automatic extraction tool that extracts structured data from text can produce a ranked list of possible extractions, each associated with a probability of being the correct one [14].

WSDs can elegantly handle such scenarios by simply adding a new column Pr to each component relation, which contains the probability for the corresponding combination of values.

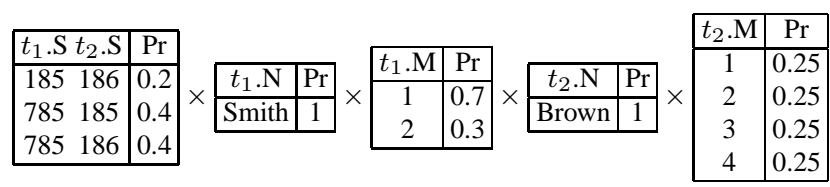

Figure 4. Probabilistic version of the WSD of Figure 3 ,

Example 1.3 Figure 4 shows a probabilistic version of the WSD of Figure 3 The probabilities in the last component imply that the possible values for the marital status of $t_{2}$ are equally likely, whereas $t_{1}$ is more likely to be single than married. The probabilities for the name values for $t_{1}$ and $t_{2}$ equal one, as this information is certain.

Given a probabilistic WSD $\left\{\mathcal{C}_{1}, \ldots, \mathcal{C}_{m}\right\}$, we obtain a possible world by choosing one tuple $w_{i}$ out of each component relation $\mathcal{C}_{i}$. The probability of this world is then computed as $\prod_{i} w_{i} . P r$. For example, in Figure 4 choosing the first, the second and the third tuple from the first, the third and the fifth component, respectively, results in the world

\begin{tabular}{c|ccc}
$\mathrm{R}$ & $\mathrm{SSN}$ & Name & MS \\
\hline$t_{1}$ & 185 & Smith & 2 \\
$t_{2}$ & 186 & Brown & 2
\end{tabular}

The world's probability can be computed as $0.2 \cdot 0.3 \cdot 0.25=$ 0.015 . 
In practice, it is often the case that fields or even tuples carry the same values in all worlds. For instance, in the census data scenario discussed above, we assumed that only one field in 10000 has several possible values. Such a world-set decomposes into a WSD in which most fields are in component relations that have precisely one tuple.

We will also consider a refinement of WSDs, WSDTs, which store information that is the same in all possible worlds once and for all in so-called template relations.

Example 1.4 The world-set of the previous examples can be represented by the WSDT of Figure 5 .

\begin{tabular}{|c|c|c|c|c|c|c|}
\hline & Temp & late & S & $\mathrm{N}$ & M & \\
\hline & $t_{1}$ & & & Smith & $?$ & \\
\hline & $t_{2}$ & & & Brow1 & ? & \\
\hline$t_{1} . \mathrm{S} t_{2} . \mathrm{S}$ & $\operatorname{Pr}$ & & & & \begin{tabular}{|l|}
$t_{2} \cdot \mathrm{M}$ \\
\end{tabular} & $\operatorname{Pr}$ \\
\hline $\begin{array}{ll}185 & 186\end{array}$ & \begin{tabular}{|l|l|}
11 \\
\end{tabular} & & $t_{1} . \mathrm{M}$ & Pr & 1 & 0.25 \\
\hline $\begin{array}{ll}785 & 185\end{array}$ & 0.4 & $x$ & 1 & $0.7 \times$ & 2 & 0.25 \\
\hline $\begin{array}{lll}785 \quad 186\end{array}$ & 0.4 & & 2 & 0.3 & 3 & 0.25 \\
\hline & & & & & 4 & 0.25 \\
\hline
\end{tabular}

\section{Figure 5. Probabilistic WSD with a template relation.}

WSDTs combine the advantages of WSDs and c-tables. In fact, WSDTs can be naturally viewed as c-tables whose formulas have been put into a normal form represented by the component relations, and null values '?' in the template relations represent fields on which the worlds disagree. Indeed, each tuple in the product of the component relations is a possible value assignment for the variables in the template relation. The following c-table with global condition $\Phi$ is equivalent to the WSDT in Figure 5 (modulo the probabilistic weights):

$$
\begin{aligned}
& \begin{array}{l|lll}
\mathrm{T} & \mathrm{S} & \mathrm{N} & \mathrm{M} \\
\hline & x & \text { Smith } & y \\
& z & \text { Brown } & w
\end{array} \\
& \Phi=((x=185 \wedge z=186) \vee(x=785 \wedge z=185) \vee \\
& (x=785 \wedge z=186)) \wedge(y=1 \vee y=2) \wedge \\
& (w=1 \vee w=2 \vee w=3 \vee w=4)
\end{aligned}
$$

The technical contributions of this article are as follows.

- We formally introduce WSDs and WSDTs and study some of their properties. Our notion is a refinement of the one presented above and allows to represent worlds over multi-relation schemas which contain relations with varying numbers of tuples. WSD(T)s can represent any finite set of possible worlds over relational databases and are therefore a strong representation system for any relational query language.
- A practical problem with WSDs and WSDTs is that a DBMS that manages such representations has to support relations of arbitrary arity: the schemata of the component relations of a decomposition depend on the data. Unfortunately, DBMS (e.g. PostgreSQL) in practice often do not support relations beyond a fixed arity.

For that reason we present refinements of the notion of WSDs, the uniform WSDs (UWSDs), and their extension by template relations, the UWSDTs, and study their properties as representation systems.

- We show how to process relational algebra queries over world-sets represented by UWSDTs. For illustration purposes, we discuss query evaluation in the context of the much more graphic WSDs.

We also develop a number of optimizations and techniques for normalizing the data representations obtained by queries to support scalable query processing even on very large world-sets.

- We describe a prototype implementation built on top of the PostgreSQL RDBMS. Our system is called MayBMS and supports the management of incomplete information using UWSDTs.

- We report on our experimental evaluation of UWSDTs as a representation system for large finite sets of possible worlds. Our experiments show that UWSDTs allow highly scalable techniques for managing incomplete information. We found that the size of UWSDTs obtained as query answers or data cleaning results remains close to that of a single world. Furthermore, the processing time for queries on UWSDTs is also comparable to processing just a single world and thus a classical relational database.

- For our experiments, we develop data cleaning techniques in the context of UWSDTs. To clean data of inconsistent worlds we chase a set of equality-generating dependencies on UWSDTs, which we brief-ly describe.

WSDs are designed to cope with large sets of worlds, which exhibit local dependencies and large commonalities. Note that this data pattern can be found in many applications. Besides the census scenario, Section 9 describes two further applications: managing inconsistent databases using minimal repairs [7, 9] and medicine data.

A fundamental assumption of this work is that one wants to manage finite sets of possible worlds. This is justified by previous work on representation systems starting with Imielinski and Lipski [15], by recent work [12, 4, 8], and by current application requirements. Our approach can deal with databases with unresolved uncertainties. Such databases are still valuable. It should be possible to do 
data transformations that preserve as much information as possible, thus necessarily mapping between sets of possible worlds. In this sense, WSDs represent a compositional framework for querying and data cleaning. A different approach is followed in, e.g., [7, 10], where the focus is on finding certain answers of queries on incomplete and inconsistent databases.

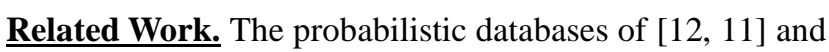
the dirty relations of [4] are examples of practical representation systems that are not strong for relational algebra. As query answers in general cannot be represented as a set of possible worlds in the same formalism, query evaluation is focused on computing the certain answers to a query, or the probability of a tuple being in the result. Such formalisms close the possible worlds semantics using clean answers [4] and probabilistic-ranked retrieval [12]. As we will see in this article, our approach subsumes the aforementioned two and is strictly more expressive than them.

In parallel to our approach, [21, 8] propose ULDBs that combine uncertainty and a low-level form of lineage to model any finite world-set. Like the dirty relations of [4], ULDBs represent a set of independent tuples with alternatives. Lineage is then used to represent dependencies among alternatives of different tuples and thus is essential for the expressive power of the formalism.

As both ULDBs and WSDs can model any finite worldset, they inherently share some similarities, yet differ in important aspects. WSDs support efficient algorithms for finding a minimal data representation based on relational factorization. Differently from ULDBs,

WSDs allow representing uncertainty at the level of tuple fields, not only of tuples. This causes, for instance, or-set relations to have linear representations as WSDs, but (in general) exponential representations as ULDBs. As reported in [8], resolving tuple dependencies, i.e., tracking which alternatives of different tuples belong to the same world, often requires to compute expensive lineage closure. Additionally, query operations on ULDBs can produce inconsistencies and anomalies, such as erroneous dependencies and inexistent tuples. In contrast, WSDs share neither of these pitfalls. As no implementation of ULDBs was available at the time of writing this document, no experimental comparison of ULDBs and WSDs could be established.

\section{Preliminaries}

We use the named perspective of the relational model with the operations selection $\sigma$, projection $\pi$, product $\times$, union $\cup$, difference - , and attribute renaming $\delta$ (cf. e.g. [2]). A relational schema is a tuple $\Sigma=$ $\left(R_{1}\left[U_{1}\right], \ldots, R_{k}\left[U_{k}\right]\right)$, where each $R_{i}$ is a relation name and $U_{i}$ is a set of attribute names. Let $\mathbf{D}$ be a set of domain elements. A relation over schema $R\left[A_{1}, \ldots, A_{k}\right]$ is a set of tuples $\left(A_{1}: a_{1}, \ldots, A_{k}: a_{k}\right)$ where $a_{1}, \ldots, a_{k} \in \mathbf{D}$. A relational database $\mathcal{A}$ over schema $\Sigma$ is a set of relations $R^{\mathcal{A}}$, one for each relation schema $R[U]$ from $\Sigma$. Sometimes, when no confusion of database may occur, we will use $R$ rather than $R^{\mathcal{A}}$ to denote one particular relation over schema $R[U]$. By the size of a relation $R$, denoted $|R|$, we refer to the number of tuples in $R$. For a relation $R$ over schema $R[U]$, let $\mathcal{S}(R)$ denote the set $U$ of its attributes and let $\operatorname{ar}(R)$ denote the arity of $R$.

A product $m$-decomposition of a relation $R$ is a set of non-nullary relations $\left\{C_{1}, \ldots, C_{m}\right\}$ such that $C_{1} \times \cdots \times$ $C_{m}=R$. The relations $C_{1}, \ldots, C_{m}$ are called components. A product $m$-decomposition of $R$ is maximal if there is no product $n$-decomposition of $R$ with $n>m$.

A set of possible worlds (or world-set) over schema $\Sigma$ is a set of databases over schema $\Sigma$. Let $\mathbf{W}$ be a set of structures, rep be a function that maps from $\mathbf{W}$ to world-sets of the same schema. Then $(\mathbf{W}$, rep $)$ is a strong representation system for a query language if, for each query $Q$ of that language and each $\mathcal{W} \in \mathbf{W}$ such that $Q$ is applicable to the worlds in $\operatorname{rep}(\mathcal{W})$, there is a structure $\mathcal{W}^{\prime} \in \mathbf{W}$ such that $\operatorname{rep}\left(\mathcal{W}^{\prime}\right)=\{Q(\mathcal{A}) \mid \mathcal{A} \in \operatorname{rep}(\mathcal{W})\}$. Obviously,

Lemma 2.1 If rep is a function from a set of structures $\mathbf{W}$ to the set of all finite world-sets, then $(\mathbf{W}$, rep) is a strong representation system for any relational query language.

\section{Probabilistic World-Set Decompositions}

In order to use classical database techniques for storing and querying incomplete data, we develop a scheme for representing a world-set $\mathbf{A}$ by a single relational database.

Let $\mathbf{A}$ be a finite world-set over schema $\Sigma=$ $\left(R_{1}, \ldots, R_{k}\right)$. For each $R$ in $\Sigma$, let $|R|_{\max }=\max \left\{\left|R^{\mathcal{A}}\right|:\right.$ $\mathcal{A} \in \mathbf{A}\}$ denote the maximum cardinality of relation $R$ in any world of $\mathbf{A}$. Given a world $\mathcal{A}$ with $R^{\mathcal{A}}=$ $\left\{t_{1}, \ldots, t_{\left|R^{\mathcal{A}}\right|}\right\}$, let $t_{R^{\mathcal{A}}}$ be the tuple obtained as the concatenation (denoted o) of the tuples of $R^{\mathcal{A}}$ in an arbitrary order padded with a special tuple $t_{\perp}=\underbrace{(\perp, \ldots, \perp)}_{a r(R)}$ up to arity $|R|_{\max }$ :

$$
t_{R^{\mathcal{A}}}:=t_{1} \circ \cdots \circ t_{\left|R^{\mathcal{A}}\right|} \circ(\underbrace{t_{\perp}, \ldots \ldots, t_{\perp}}_{|R|_{\max }-\left|R^{\mathcal{A}}\right|})
$$

Then tuple $t_{\mathcal{A}}:=t_{R_{1}^{\mathcal{A}}} \circ \cdots \circ t_{R_{k}^{\mathcal{A}}}$ encodes all the information in world $\mathcal{A}$. The "dummy" tuples with $\perp$-values are only used to ensure that the relation $R$ has the same number of tuples in all worlds in $\mathbf{A}$. We extend this interpretation and generally define as $t_{\perp}$ any tuple that has at least one symbol $\perp$, i.e., $\left(A_{1}: a_{1}, \ldots, A_{n}: a_{n}\right)$, where at least one $a_{i}$ is $\perp$, is a $t_{\perp}$ tuple. This allows for several different inlinings of the same world-set. 
By a world-set relation of a world-set $\mathbf{A}$, we denote the relation $\left\{t_{\mathcal{A}} \mid \mathcal{A} \in \mathbf{A}\right\}$. This world-set relation has schema $\left\{R . t_{i} .\left.A_{j}|R[U] \in \Sigma, 1 \leq i \leq| R\right|_{\max }, A_{j} \in U\right\}$. Note that in defining this schema we use $t_{i}$ to denote the position (or identifier) of tuple $t_{i}$ in $t_{R^{\mathcal{A}}}$ and not its value.

Given the above definition that turned every world in a tuple of a world-set relation, computing the initial world-set is an easy exercise. In order to have every world-set relation define a world-set, let a tuple extracted from some $t_{R^{\mathcal{A}}}$ be in $R^{\mathcal{A}}$ iff it does not contain any occurrence of the special symbol $\perp$. That is, we map $t_{R^{\mathcal{A}}}=\left(a_{1}, \ldots, a_{\operatorname{ar}(R) \cdot|R|_{\max }}\right)$ to $R^{\mathcal{A}}$ as

$$
\begin{gathered}
t_{R^{\mathcal{A}}} \mapsto\left\{\left.\left(a_{\operatorname{ar}(R) \cdot k+1}, \ldots, a_{a r(R) \cdot(k+1)}\right)|0 \leq k<| R\right|_{\max }\right. \\
\left.a_{a r(R) \cdot k+1} \neq \perp, \ldots, a_{a r(R) \cdot(k+1)} \neq \perp\right\} .
\end{gathered}
$$

Observe that although world-set relations are not unique as we have left open the ordering in which the tuples of a given world are concatenated, all world-set relations of a world-set $\mathbf{A}$ are equally good for our purposes because they can be mapped invariantly back to $\mathbf{A}$. Note that for each world-set relation a maximal decomposition exists, is unique, and can be efficiently computed [6].

Definition 3.1 Let A be a world-set and $W$ a world-set relation representing $\mathbf{A}$. Then a world-set $m$-decomposition $(m-W S D)$ of $\mathbf{A}$ is a product $m$-decomposition of $W$.

We will refer to each of the $m$ elements of a world-set $m$ decomposition as components, and to the component tuples as local worlds. Somewhat simplified examples of worldset relations and WSDs over a single relation $R$ (thus " $R$ " was omitted from the attribute names of the world-set relations) were given in Section 1] Further examples can be found in Section 4 It should be emphasized that with WSDs we can also represent multiple relational schemata and even components with fields from different relations.

It immediately follows from our definitions that

Proposition 3.2 Any finite set of possible worlds can be represented as a world-set relation and as a 1-WSD.

Corollary 3.3 (Lemma 2.1) WSDs are a strong representation system for any relational query language.

As pointed out in Section 1, this is not true for or-set relations. For the relatively small class of world-sets that can be represented as or-set relations, the size of our representation system is linear in the size of the or-set relations. As seen in the examples, our representation is much more space-efficient than world-set relations.

Modeling Probabilistic Information. We can quantify the uncertainty of the data by means of probabilities using a natural extension of WSDs. A probabilistic worldset m-decomposition (probabilistic m-WSD) is an m-WSD

$\left\{\mathcal{C}_{1}, \ldots, \mathcal{C}_{m}\right\}$, where each component relation $\mathcal{C}$ has a special attribute $\mathrm{Pr}$ in its schema defining the probability for the local worlds, that is, for each combination of values defined by the component. We require that the probabilities in a component sum up to one, i.e. $\sum_{t_{C} \in \mathcal{C}} t_{C} \cdot \operatorname{Pr}=1$.

Probabilistic WSDs generalize the probabilistic tupleindependent model of [12], as we show next. Figure 6 (a) is an example taken from [12]. It shows a probabilistic database with two relations $S$ and $T$. Each tuple is assigned a confidence value, which represents the probability of the tuple being in the database, and the tuples are assumed independent. A possible world is obtained by choosing a subset of the tuples in the probabilistic database, and its probability is computed by multiplying the probabilities for selecting a tuple or not, depending on whether that tuple is in the world. The set of possible worlds for $D$ is given in Figure 6(b). For example, the probability of the world $D_{3}$ can be computed as $(1-0.2) \cdot 0.5 \cdot 0.6=0.06$.

\begin{tabular}{c|cc|c}
$\mathrm{S}$ & $\mathrm{A}$ & $\mathrm{B}$ & $\mathrm{Pr}$ \\
\hline$s_{1}$ & $\mathrm{~m}$ & 1 & 0.8 \\
$s_{2}$ & $\mathrm{n}$ & 1 & 0.5 \\
$\mathrm{~T}$ & $\mathrm{C}$ & $\mathrm{D}$ & $\mathrm{Pr}$ \\
\hline$t_{1}$ & 1 & $\mathrm{p}$ & 0.6
\end{tabular}

(a)

\begin{tabular}{|l|c} 
world & $\operatorname{Pr}$ \\
\hline$D_{1}=\left\{s_{1}, s_{2}, t_{1}\right\}$ & 0.24 \\
$D_{2}=\left\{s_{1}, t_{1}\right\}$ & 0.24 \\
$D_{3}=\left\{s_{2}, t_{1}\right\}$ & 0.06 \\
$D_{4}=\left\{t_{1}\right\}$ & 0.06 \\
$D_{5}=\left\{s_{1}, s_{2}\right\}$ & 0.16 \\
$D_{6}=\left\{s_{1}\right\}$ & 0.16 \\
$D_{7}=\left\{s_{2}\right\}$ & 0.04 \\
$D_{8}=\emptyset$ & 0.04
\end{tabular}

(b)

\section{Figure 6. A probabilistic database for rela- tions $S$ and $T(a)$, and the represented set of possible worlds (b).}

We obtain a probabilistic WSD in the following way. Each tuple $t$ with confidence $c$ in a probabilistic database induces a WSD component representing two local worlds: the local world with tuple $t$ and probability $c$, and the empty world with probability $1-c$. Figure 7 gives the WSD encoding of the probabilistic database of Figure 6 Of course, in probabilistic WSDs we can assign probabilities not only to individual tuples, but also to combinations of values for fields of different tuples or relations.

\begin{tabular}{|c|cc|c|}
\hline$C_{1}$ & $s_{1} . \mathrm{A}$ & $s_{1} . \mathrm{B}$ & $\mathrm{Pr}$ \\
\hline 1 & $\mathrm{~m}$ & 1 & 0.8 \\
2 & $\perp$ & $\perp$ & 0.2
\end{tabular}$\times$\begin{tabular}{|c|cc|c|}
\hline$C_{2}$ & $s_{2} . \mathrm{A}$ & $s_{2} . \mathrm{B}$ & $\mathrm{Pr}$ \\
\hline 1 & $\mathrm{n}$ & 1 & 0.5 \\
2 & $\perp$ & $\perp$ & 0.5 \\
\hline
\end{tabular}$\times$\begin{tabular}{|c|c|cc|c|}
\hline$C_{3}$ & $t_{1} . \mathrm{C} t_{1} . \mathrm{D}$ & $\mathrm{Pr}$ \\
\hline 1 & 1 & $\mathrm{p}$ & 0.6 \\
2 & $\perp$ & $\perp$ & 0.4 \\
\hline
\end{tabular}

Figure 7. WSD equivalent to the probabilistic database in Figure 6(a). 
Adding Template Relations. We now present our refinement of WSDs with so-called template relations. A template stores information that is the same in all possible worlds and contains special values '?' $\notin \mathbf{D}$ in fields at which different worlds disagree.

Let $\Sigma=\left(R_{1}, \ldots, R_{k}\right)$ be a schema and $\mathbf{A}$ a finite set of possible worlds over $\Sigma$. Then, the database $\left(R_{1}^{0}, \ldots, R_{k}^{0},\left\{C_{1}, \ldots, C_{m}\right\}\right)$ is called an $m$-WSD with template relations ( $m$-WSDT) of $\mathbf{A}$ iff there is a WSD $\left\{C_{1}, \ldots, C_{m}, D_{1}, \ldots, D_{n}\right\}$ of $\mathbf{A}$ such that $\left|D_{i}\right|=1$ for all $i$ and if relation $D_{i}$ has attribute $R_{j}$.t. $A$ and value $v$ in its unique $R_{j}$.t.A-field, then the template relation $R_{j}^{0}$ has a tuple with identifier $t$ whose $A$-field has value $v$.

Of course WSDTs again can represent any finite worldset and are thus a strong representation system for any relational query language. Example 1.4 shows a WSDT for the running example of the introduction.

Uniform World-Set Decompositions. In practice database systems often do not support relations of arbitrary arity (e.g., WSD components). For that reason we introduce next a modified representation of WSDs called uniform WSDs. Instead of having a variable number of component relations, possibly with different arities, we store all values in a single relation $C$ that has a fixed schema. We use the fixed schema consisting of the three relation schemata

$C[F I D, L W I D, V A L], F[F I D, C I D], W[C I D, L W I D, P R]$

where FID is a triple1 (Rel, TupleID, Attr) denoting the Attr-field of tuple TupleID in database relation Rel.

In this representation we need a restricted flavor of world-ids called local world-ids (LWIDs). The local worldids refer only to the possible worlds within one component. LWIDs avoid the drawbacks of "global" world IDs for the individual worlds. This is important, since the size of global world IDs can exceed the size of the decomposition itself, thus making it difficult or even impossible to represent the world-sets in a space-efficient way. If any world-set over a given schema and a fixed active domain is permitted, one can verify that global world-ids cannot be smaller than the largest possible world over the schema and the active domain.

Given a WSD $\left\{C_{1}, \ldots, C_{m}\right\}$ with schemata $C_{i}\left[U_{i}\right]$, we populate the corresponding UWSD as follows.

- $((R, t, A), s, v) \in C$ iff, for some (unique) $i$, R.t.A $\in$ $U_{i}$ and the field of column R.t.A in the tuple with id $s$ of $C_{i}$ has value $v$.

- $F:=\left\{\left((R, t, A), C_{i}\right) \mid 1 \leq i \leq m\right.$, R.t. $\left.A \in U_{i}\right\}$,

- $\left(C_{i}, s, p\right) \in W$ iff there is a tuple with identifier $s$ in $C_{i}$, whose probability is $p$.

\footnotetext{
${ }^{1}$ That is, FID really takes three columns, but for readability we keep them together under a common name in this section.
}

\begin{tabular}{|c|c|c|c|c|c|c|c|}
\hline$R^{0}$ & $\mathrm{~S}$ & $\mathrm{~N}$ & $\mathrm{M}$ & & \multirow{2}{*}{\multicolumn{2}{|c|}{ FID }} & \\
\hline$t_{1}$ & $?$ & Smith & $?$ & $\mathrm{~F}$ & & & CID \\
\hline$t_{2}$ & $?$ & Brown & 3 & & \multicolumn{2}{|c|}{$\begin{array}{c}\left(R, t_{1}, S\right) \\
\left(R, t_{1}, M\right)\end{array}$} & $\begin{array}{l}C_{1} \\
C_{2}\end{array}$ \\
\hline $\mathrm{C}$ & FID & LWID & VAL & & \multicolumn{2}{|c|}{$\left(R, t_{2}, S\right)$} & $C_{1}$ \\
\hline & $\left(R, t_{1}, S\right)$ & 1 & 185 & & & & \\
\hline & $\left(R, t_{2}, S\right)$ & 1 & 186 & W & CID & LWID & PR \\
\hline & $\left(R, t_{1}, S\right)$ & 2 & 785 & & $C_{1}$ & 1 & $\overline{0.2}$ \\
\hline & $\left(R, t_{2}, S\right)$ & 2 & 185 & & $C_{1}$ & 2 & 0.4 \\
\hline & $\left(R, t_{1}, S\right)$ & 3 & 785 & & $C_{1}$ & 3 & 0.4 \\
\hline & $\left(R, t_{2}, S\right)$ & 3 & 186 & & $C_{2}$ & 1 & 0.7 \\
\hline & $\left(R, t_{1}, M\right)$ & 1 & 1 & & $C_{2}$ & 2 & 0.3 \\
\hline & $\left(R, t_{1}, M\right)$ & 2 & 2 & & & & \\
\hline
\end{tabular}

\section{Figure 8. A UWSDT corresponding to the WSDT of Figure 5 .}

Intuitively, the relation $C$ stores each value from a component together with its corresponding field identifier and the identifier of the component-tuple in the initial WSD (column $L W I D$ of $C$ ). The relation $F$ contains the mapping between tuple fields and component identifiers, and $W$ keeps track of the worlds present for a given component.

In general, the VAL column in the component relation C must store values for fields of different type. One possibility is to store all values as strings and use casts when required. Alternatively, one could have one component relation for each data type. In both cases the schema remains fixed.

Finally, we add template relations to UWSDs in complete analogy with WSDTs, thus obtaining the UWSDTs.

Example 3.4 We modify the world-set represented in Figure 4 such that the marital status in $t_{2}$ can only have the value 3 . Figure 8 is then the uniform version of the WSDT of Figure 4. Here $R^{0}$ contains the values that are the same in all worlds. For each field that can have more than one possible value, $R^{0}$ contains a special placeholder, denoted by '?'. The possible values for the placeholders are defined in the component table $C$. In practice, we can expect that the majority of the data fields can take only one value across all worlds, and can be stored in the template relation.

Proposition 3.5 Any finite set of possible worlds can be represented as a 1-UWSD and as a 1-UWSDT.

It follows again that UWSD(T)s are a strong representation system for any relational query language.

\section{Queries on World-set Decompositions}

In this section we study the query evaluation problem for WSDs. As pointed out before, UWSDTs are a better representation system than WSDs; nevertheless WSDs are sim- 
pler to explain and visualize and the main issues regarding query evaluation are the same for both systems.

The goal of this section is to provide, for each relational algebra query $Q$, a query $\hat{Q}$ such that for a $\operatorname{WSD} \mathcal{W}$,

$$
\operatorname{rep}(\hat{Q}(\mathcal{W}))=\{Q(\mathcal{A}) \mid \mathcal{A} \in \operatorname{rep}(\mathcal{W})\} .
$$

Of course we want to evaluate queries directly on WSDs using $\hat{Q}$ rather than process the individual worlds using the original query $Q$.

The algorithms for processing relational algebra que-ries presented next are orthogonal to whether or not the WSD stores probabilities. According to our semantics, a query is conceptually evaluated in each world and extends the world with the result of the query in that world. A different class of queries are those that close the possible world semantics and compute confidence of tuples in the result of a query. This will be the subject of Section 6

When compared to traditional query evaluation, the evaluation of relational queries on WSDs poses new challenges. First, since decompositions in general consist of several components, a query $\hat{Q}$ that maps from one WSD to another must be expressed as a set of queries, each of which defines a different component of the output WSD. Second, as certain query operations may cause new dependencies between components to develop, some components may have to be merged (i.e., part of the decomposition undone using the product operation $\times$ ). Third, the answer to a (sub)query $Q_{0}$ must be represented within the same decomposition as the input relations; indeed, we want to compute a decomposition of world set $\left\{\left(\mathcal{A}, Q_{0}(\mathcal{A})\right) \mid \mathcal{A} \in \operatorname{rep}(\mathcal{W})\right\}$ in order to be able to resort to the input relations as well as the result of $Q_{0}$ within each world. Consider for example a query $\sigma_{A=1}(R) \cup \sigma_{B=2}(R)$. If we first compute $\sigma_{A=1}(R)$, we must not replace $R$ by $\sigma_{A=1}(R)$, otherwise $R$ will not be available for the computation of $\sigma_{B=2}(R)$. On the other hand, if $\sigma_{A=1}(R)$ is stored in a separate WSD, the connection between worlds of $R$ and the selection $\sigma_{A=1}$ is lost and we can again not compute $\sigma_{A=1}(R) \cup \sigma_{B=2}(R)$.

We say that a relation $P$ is a copy of another relation $R$ in a WSD if $R$ and $P$ have the same tuples in every world represented by the WSD. For a component $C$, an attribute R.t. $A_{i}$ of $C$ and a new attribute P.t.B, the function ext extends $C$ by a new column P.t.B that is a copy of R.t. $A_{i}$ :

$$
\begin{aligned}
\operatorname{ext}\left(C, A_{i}, B\right):= & \left(A_{1}: a_{1}, \ldots, A_{n}: a_{n}, B: a_{i}\right) \mid \\
& \left.\left(A_{1}: a_{1}, \ldots, A_{n}: a_{n}\right) \in C\right\}
\end{aligned}
$$

Then $\operatorname{copy}(R, P)$ executes $C:=\operatorname{ext}\left(C\right.$, R.t $\left._{i} . A, P . t_{i} . A\right)$ for each component $C$ and each $R . t_{i} . A \in \mathcal{S}(C)$.

The implementation of some operations requires the composition of components. Let $C_{1}$ and $C_{2}$ be two components with schemata $\left(A_{1}, \ldots, A_{k}, \operatorname{Pr}\right)$, and $\left(B_{1}, \ldots, B_{l}, P r\right)$, respectively. Then the composition of $C_{1}$ and $C_{2}$ is defined as:

$$
\begin{aligned}
\text { compose } & \left(C_{1}, C_{2}\right):= \\
\{ & \left(A_{1}: a_{1}, \ldots, A_{k}: a_{k}, B_{1}: b_{1}, \ldots, B_{l}: b_{l},\right. \\
& \left.\operatorname{Pr}: p_{1} \cdot p_{2}\right) \mid \\
& \left(A_{1}: a_{1}, \ldots, A_{k}: a_{k}, \operatorname{Pr}: p_{1}\right) \in C_{1}, \\
& \left.\left(B_{1}: b_{1}, \ldots, B_{l}: b_{l}, \operatorname{Pr}: p_{2}\right) \in C_{2}\right\}
\end{aligned}
$$

In the non-probabilistic case the composition of components is simply the relational product of the two components.

Figure 9 presents implementations of the relational algebra operations selection (of the form $\sigma_{A \theta c}$ or $\sigma_{A \theta B}$, where $A$ and $B$ are attributes, $c$ is a constant, and $\theta$ is a comparison operation, $=, \neq,<, \leq,>$, or $\geq$ ), projection, relational product and union on WSDs. In each case, the input WSD is extended by the result of the operation.

Let us now have a closer look at the evaluation of relational algebra operations on WSDs. For this, we use as running example the set of eight worlds over the relation $R$ of Figure 10 (a) and its maximal 7-WSD of Figure10 (b). The second component (from the left) of the WSD spans over several tuples and attributes and each of the remaining six components refer to one tuple and one attribute. The first tuple of the second component of the WSD of Figure 10 contains the values for $R . t_{1} . B, R . t_{1} . C$, and $R . t_{2} . B$, i.e. some but not all of the attributes of the first and second tuple of $R^{\mathcal{A}}$, for all worlds $\mathcal{A}$. Because of space limitations and our attempt to keep the WSDs readable, we consistently show in the following examples only the WSDs of the result relations.

Selection with condition $A \theta c$. In order to compute a selection $P:=\sigma_{A \theta c}(R)$, we first compute a copy $P$ of relation $R$ and subsequently drop tuples of $P$ that do not match the selection condition.

Dropping tuples is a fairly subtle operation, since tuples can spread over several components and a component can define values for more than one tuple.

Thus a selection must not delete tuples from component relations, but should mark fields as belonging to deleted tuples using the special value $\perp$. To evaluate $\sigma_{A \theta c}(R)$, our selection algorithm of Figure 9 checks for each tuple $t_{i}$ in the relation $P$ and $t_{C}$ in component $C$ with attribute $P . t_{i} . A$ whether $t_{C} .\left(P . t_{i} . A\right) \theta c$. In the negative case the tuple P.t is marked as deleted in all worlds that take values from $t_{C}$. For that, $t_{C}$. $\left(P . t_{i} . A\right)$ is assigned value $\perp$, and all other attributes $P . t_{i} . A^{\prime}$ of $C$ referring to the same tuple $t_{i}$ of $P$ are assigned value $\perp$ in $t_{C}$, (cf. the algorithm propagate- $\perp$ of Figure 12). This assures that if we later project away the attribute $A$ of $P$, we do not erroneously "reintroduce" tuple $P . t_{i}$ into worlds that take values from $t_{C}$.

Example 4.1 Figure 11 shows the answers to $\sigma_{C=7}(R)$ and $\sigma_{B=1}(R)$. Note that the resulting WSDs should contain 


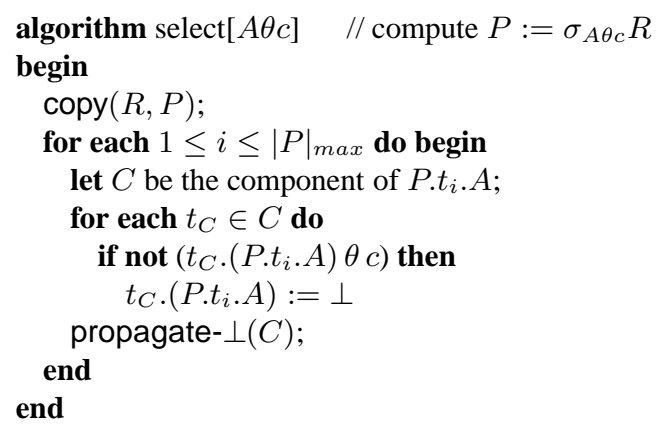

algorithm project $[U] \quad / /$ compute $P:=\pi_{U}(R)$

begin

$\operatorname{copy}(R, P)$;

for each $1 \leq i \leq|P|_{\max }$ do

while no fixpoint is reached do begin

let $C$ be the component of P.t..$A$, where $A \in U$;

let $C^{\prime} \neq C$ be the component of P.t. $t_{i}$, where

$B \notin U$ and $\left(\forall A^{\prime} \in U: P . t_{i} . A^{\prime} \notin \mathcal{S}\left(C^{\prime}\right)\right)$ and

$\left(\exists t_{C^{\prime}} \in C^{\prime}: t_{C^{\prime}} \cdot B=\perp\right)$;

replace components $C, C^{\prime}$ by $C:=\operatorname{compose}\left(C, C^{\prime}\right)$;

propagate- $\perp(C)$;

project away $P . t_{j} . B$ from $C$ where $B \notin U$ and $j \leq i$; end

for each $1 \leq i \leq|P|_{\max }$ and $B \notin U$ do begin

let $C$ be the component of P.t. $B$;

project away $P . t_{i} . B$ from $C$;

end

end

algorithm rename // compute $\delta_{A \rightarrow A^{\prime}}(R)$

begin

for each $1 \leq i \leq|R|_{\max }$ do begin

let $C$ be the component of R.t. $t_{i}$;

$C:=\delta_{R \cdot t_{i} \cdot A \rightarrow R \cdot t_{i} \cdot A^{\prime}}(C)$;

end;

end

algorithm difference $\quad / /$ compute $P:=R-S$

begin

$\operatorname{copy}(R, P)$;

for each $1 \leq i \leq|P|_{\max }$ do

for each $1 \leq j \leq|S|_{\max }$ do

let $C_{1}, \ldots, C_{k}$ be the components for the fields of P.t $t_{i}$ and $S . t_{j}$; replace $C_{1}, \ldots, C_{k}$ by $C:=\operatorname{compose}\left(C_{1}, \ldots, C_{k}\right)$;

for each $t_{C} \in C$ do begin

if $t_{C} \cdot\left(P . t_{i} \cdot A\right)=t_{C} \cdot\left(S . t_{j} . A\right)$ for all $A \in S(R)$ then $t_{C} \cdot\left(P . t_{i} \cdot A\right):=\perp$;

end

end

end

Figure 9. Evaluating relational algebra operations on WSDs. 


\begin{tabular}{|c|c|c|c|c|c|c|c|c|}
\hline$\overline{\mathrm{A}}$ & B C & $\mathrm{A} B \mathrm{C}$ & A B C & $\mathrm{A} \quad \mathrm{B} \quad \mathrm{C}$ & $\mathrm{A} \quad \mathrm{B} \quad \mathrm{C}$ & A B C & $\mathrm{A} \quad \mathrm{B} \quad \mathrm{C}$ & A B C \\
\hline & 10 & $\begin{array}{lll}2 & 1 & 0\end{array}$ & $\begin{array}{lll}1 & 1 & 0\end{array}$ & $\begin{array}{lll}2 & 1 & 0\end{array}$ & $\begin{array}{lll}1 & 2 & 7\end{array}$ & $\begin{array}{lll}2 & 2 & 7\end{array}$ & $\begin{array}{lll}1 & 2 & 7\end{array}$ & $\begin{array}{lll}2 & 2 & 7\end{array}$ \\
\hline 4 & 30 & $\begin{array}{lll}4 & 3 & 0\end{array}$ & $\begin{array}{lll}5 & 3 & 0\end{array}$ & $\begin{array}{lll}5 & 3 & 0\end{array}$ & $\begin{array}{lll}4 & 4 & 0\end{array}$ & $\begin{array}{lll}4 & 4 & 0\end{array}$ & $\begin{array}{lll}5 & 4 & 0\end{array}$ & $\begin{array}{lll}5 & 4 & 0\end{array}$ \\
\hline 6 & 67 & $\begin{array}{lll}6 & 6 & 7\end{array}$ & $\begin{array}{lll}6 & 6 & 7\end{array}$ & $\begin{array}{lll}6 & 6 & 7\end{array}$ & $\begin{array}{lll}6 & 6 & 7\end{array}$ & $\begin{array}{lll}6 & 6 & 7\end{array}$ & $\begin{array}{lll}6 & 6 & 7\end{array}$ & $\begin{array}{lll}6 & 6 & 7\end{array}$ \\
\hline
\end{tabular}

(a) Set of eight worlds of the relation $R$.

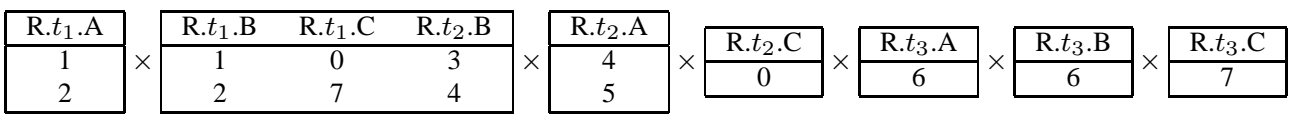

(b) 7-WSD of the world-set of (a).

Figure 10. World-set and its decomposition.

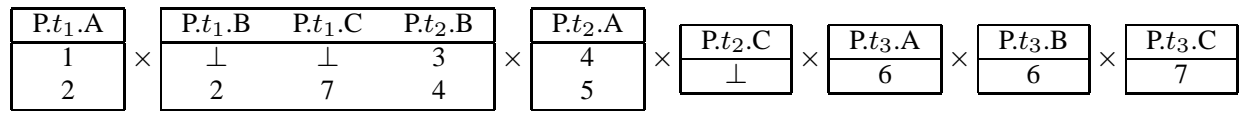

(a) $P:=\sigma_{C=7}(R)$ applied to the WSD of Figure 10

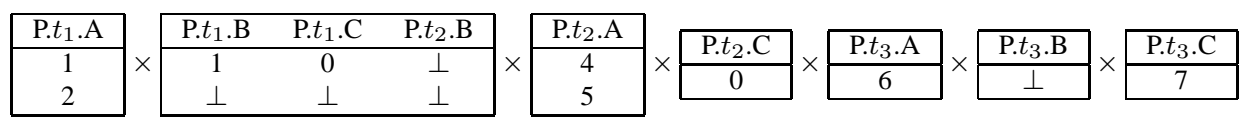

(b) $P:=\sigma_{B=1}(R)$ applied to the WSD of Figure 10

Figure 11. Selections $P:=\sigma_{C=7}(R)$ and $P:=\sigma_{B=1}(R)$ with $R$ from Figure 10 ,

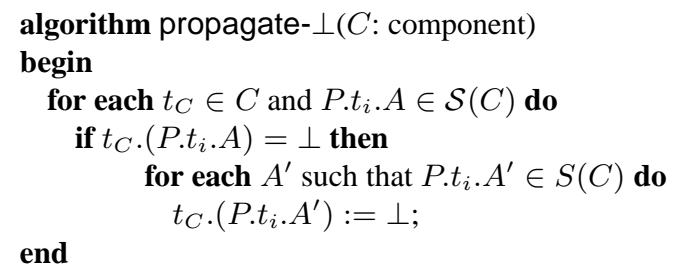

Figure 12. Propagating $\perp$-values.

both the query answer $P$ and the original relation $R$, but due to space limitations we only show the representation of $P$. One can observe that for both results in Figure 11 we obtain worlds of different sizes. For example the worlds that take values from the first tuple of the second component relation in Figure 11 (a) do not have a tuple $t_{1}$, while the worlds that take values from the second tuple of that component relation contain $t_{1}$.

Selection with condition $A \theta B$. The main added difficulty of selections with conditions $A \theta B$ as compared to selections with conditions $A \theta c$ is that it creates dependencies between two attributes of a tuple, which do not necessarily reside in the same component.

As the current decomposition may not capture exactly the combinations of values satisfying the join condition, components that have values for $A$ and $B$ of the same tuple are composed. After the composition phase, the selection algorithm follows the pattern of the selection with constant.
Example 4.2 Consider the query $\sigma_{A=B}(R)$, where $R$ is represented by the 7-WSD of Figure 10. Figure 13 shows the query answer, which is a 4-WSD that represents five worlds, where one world has three tuples, three worlds have two tuples each, and one world has one tuple.

Product. The product $T:=R \times S$ of two relations $R$ and $S$, which have disjunct attribute sets and are represented by a WSD requires that the product relation $T$ extends a component $C$ with $|S|_{\max }$ (respectively $|R|_{\max }$ ) copies of each column of $C$ with values of $R$ (respectively $S$ ). Additionally, the $i$ th $\left(j\right.$ th) copy is named $T . t_{i j} . A$ if the original has name $R . t_{i} . A$ or $S . t_{j} . A$.

Example 4.3 Figure 14 (b) shows the WSD for the product of relations $R$ and $S$ represented by the WSD of Figure 14 (a). To save space, the relations $R$ and $S$ have been removed from Figure 14 (b), and attribute names do not show the relation name " $T$ ".

Projection. A projection $P=\pi_{U}(R)$ on an attribute set $\bar{U}$ of a relation $R$ represented by the WSD $\mathcal{C}$ is translated into (1) the extension of $\mathcal{C}$ with the copy $P$ of $R$, and (2) projections on the components of $\mathcal{C}$, where all component attributes that do not refer to attributes of $P$ in $U$ are discarded. Before removing attributes, however, we need to propagate $\perp$-values, as discussed in the following example.

Example 4.4 Consider the 3-WSD of Figure 15 (a) representing a set of two worlds for $R$, where one world contains only the tuple $t_{1}$ and the other contains only the tuple $t_{2}$. Let 


\begin{tabular}{|ccccc|}
\hline P. $t_{1} . \mathrm{A}$ & $\mathrm{P} . t_{1} . \mathrm{B}$ & $\mathrm{P} . t_{1} . \mathrm{C}$ & $\mathrm{P} . t_{2} . \mathrm{A}$ & $\mathrm{P} . t_{2} . \mathrm{B}$ \\
\hline 1 & 1 & 0 & $\perp$ & $\perp$ \\
$\perp$ & $\perp$ & $\perp$ & $\perp$ & $\perp$ \\
$\perp$ & $\perp$ & $\perp$ & 4 & 4 \\
2 & 2 & 7 & 4 & 4 \\
2 & 2 & 7 & $\perp$ & $\perp$ \\
\hline
\end{tabular}

Figure 13. $P=\sigma_{A=B}(R)$ with $R$ from Figure 10.

\begin{tabular}{|c|c|c|c|c|c|c|c|c|c|c|c|c|}
\hline R. $t_{1} \cdot \mathrm{A}$ & & R. $t_{1} \cdot \mathrm{B}$ & R. $t_{2} \cdot \mathrm{A}$ & & R. $t_{2} \cdot \mathrm{B}$ & & S. $t_{1} \cdot \mathrm{C}$ & & S. $t_{1} . \mathrm{D}$ & S.t. $t_{2} \cdot \mathrm{C}$ & & S. $t_{2} . \mathrm{D}$ \\
\hline $\begin{array}{l}1 \\
2\end{array}$ & $x$ & $\begin{array}{l}3 \\
4\end{array}$ & $\begin{array}{l}5 \\
6\end{array}$ & $x$ & $\begin{array}{l}7 \\
8\end{array}$ & $x$ & $\begin{array}{l}\mathrm{a} \\
\mathrm{b}\end{array}$ & $x$ & $\begin{array}{l}\mathrm{c} \\
\mathrm{d}\end{array}$ & $\begin{array}{l}\mathrm{e} \\
\mathrm{f}\end{array}$ & $x$ & $\begin{array}{l}\mathrm{g} \\
\mathrm{h}\end{array}$ \\
\hline
\end{tabular}

(a) WSD of two relations $R$ and $S$.

\begin{tabular}{|c|c|c|c|c|c|c|c|c|c|c|c|c|c|c|c|c|c|c|c|}
\hline$t_{11 .}$ & 12. A & \multirow{3}{*}{$x$} & $t_{11 .}$ & 12. & 21. & $22 . \mathrm{A}$ & \multirow{3}{*}{$\times$} & \multicolumn{2}{|c|}{$t_{21} . \mathrm{B} t_{22} . \mathrm{B}$} & \multirow{3}{*}{$x$} & \multicolumn{2}{|c|}{$t_{11} \cdot \mathrm{C} t_{21} \cdot \mathrm{C}$} & \multirow{3}{*}{$\times$} & \multicolumn{4}{|c|}{$t_{11} \cdot \mathrm{D} t_{21} \cdot \mathrm{D} t_{12} \cdot \mathrm{C} t_{22} \cdot \mathrm{C}$} & & $t_{12} . \mathrm{D} t_{22} . \mathrm{D}$ \\
\hline 1 & 1 & & 3 & 3 & 5 & 5 & & 7 & 7 & & a & $\mathrm{a}$ & & $\mathrm{c}$ & $\mathrm{c}$ & $\mathrm{e}$ & $\mathrm{e}$ & $x$ & $\mathrm{~g}$ \\
\hline 2 & 2 & & 4 & 4 & 6 & 6 & & 8 & 8 & & b & b & & $\mathrm{d}$ & d & f & $\mathrm{f}$ & & $\mathrm{h}$ \\
\hline
\end{tabular}

(b) WSD of their product $R \times S$.

Figure 14. The product operation $R \times S$.

$P^{\prime}$ represent the first two components of $R$, which contain all values for the attribute $A$ in both tuples. The relation $P^{\prime}$ is not the answer to $\pi_{A}(R)$, because it encodes one world with both tuples, and the information from the third component of $R$ that only one tuple appears in each world is lost. To compute the correct answer, we progressively (1) compose the components referring to the same tuple (in this case all three components), (2) propagate $\perp$-values within the same tuple, and (3) project away the irrelevant attributes. The correct answer $P$ is given in Figure 15(b).

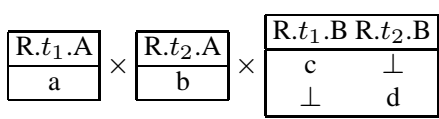

(a) WSD for R.

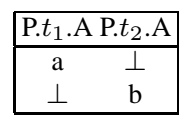

(a) WSD for P.
Figure 15. Projection $P:=\pi_{A}(R)$.

The algorithm for projection is given in Figure 9. For each tuple $t_{i}$, attribute $A$ in the projection list, and attribute $B$ not in the projection list, the algorithm first propagates the $\perp$-values of $P . t_{i} . B$ of component $C^{\prime}$ to $P . t_{i} . A$ of component $C$. If $C$ and $C^{\prime}$ are the same, the propagation is done locally within the component. Otherwise, $C$ and $C^{\prime}$ are merged before the propagation. Note that the propagation is only needed if some tuples of $C^{\prime}$ have at $\perp$-value for $t_{i}$. B. This procedure is performed until no other components $C$ and $C^{\prime}$ exist that satisfy the above criteria. After the propagation phase, the attributes not in the projection list are dropped from all remaining components.

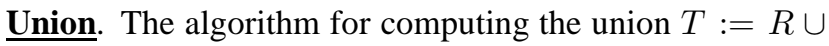
$S$ of two relations $R$ and $S$ works similarly to that for the product. Each component $C$ containing values of $R$ or $S$ is extended such that in each world of $C$ all values of $R$ and $S$ become also values of $T$.

Renaming. The operation $\delta_{A \rightarrow A^{\prime}}(R)$ renames attribute $A$ of relation $R$ to $A^{\prime}$ by renaming all attributes R.t.A in a component $C$ to R.t. $A^{\prime}$.

Difference. To compute the difference operation $P:=$ $R-S$ we scan and compose components of the two relations $R$ and $S$. For the worlds where a tuple $t$ from $R$ matches some tuple from $S$, we place $\perp$-values to denote that $t$ is not in these worlds of $P$; otherwise $t$ becomes a tuple of $P$. The difference is by far the least efficient operation to implement, as it can lead to the composition of all components in the WSD.

\section{Efficient Query Evaluation on UWSDTs}

The algorithms for computing the relational operations on WSDs presented in Section 4 can be easily adapted to UWSDTs. To do this, we follow closely the mapping of WSDs, represented as sets of components $\mathcal{C}$, to equivalent UWSDTs, represented by a triple $(F, C, W)$ and at least one template relation $R^{0}$ :

- Consider a component $K$ of WSD $\mathcal{C}$ having an attribute R.t.A with a value $v$. In the equivalent UWSDT, this value can be stored in the template relation $R^{0}$ if $v$ is the only value of R.t.A, or in the component $C$ otherwise. In the latter case, the template $R^{0}$ contains the placeholder R.t.A in the tuple $t$. In addition, in the mapping relation $F$ there is an entry with the placeholder R.t.A and a component identifier $c$, and $C$ contains a tuple formed by R.t.A, the value $v$ and a world identifier $w$.

- Worlds of different sizes are represented in WSDs by 
allowing $\perp$ values in components, and in UWSDTs by allowing for a same placeholder different amount of values in different worlds.

Any relational query is rewritten in our framework to a sequence of SQL queries, except for the projection and selection with join conditions, where the fixpoint computations are encoded as recursive PL/SQL programs. In all cases, the size of the rewriting is linear in the size of the input query. Figure 16 shows the implementation of the selection with constant on UWSDTs.

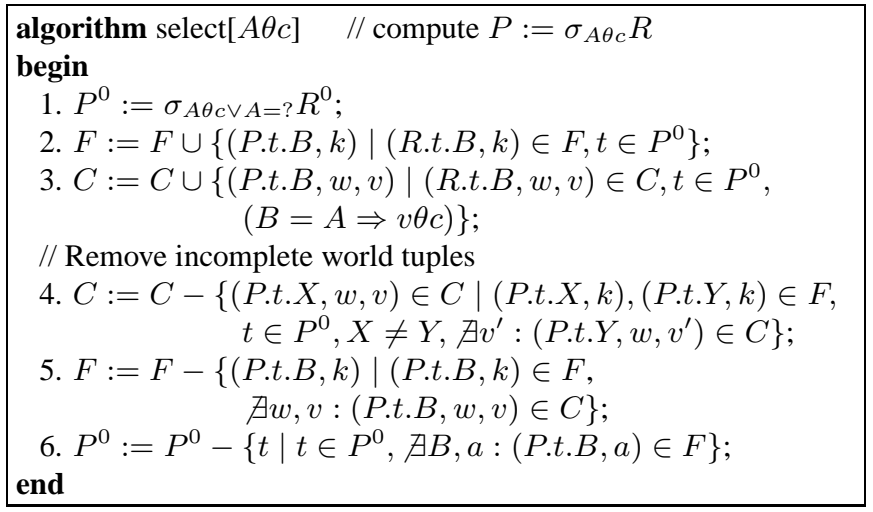

Figure 16. Evaluating $P:=\sigma_{A \theta c}(R)$ on UWSDTs.

In contrast to some algorithms of Figure 9, for UWSDTs we do not create a copy $P$ of $R$ at the beginning, but rather compute directly $P$ from $R$ using standard relational algebra operators. The template $P^{0}$ is initially the set of tuples of $R^{0}$ that satisfy the selection condition, or have a placeholder '?' for the attribute $A$ (line 1). We extend the mapping relation $F$ with the placeholders of $P^{0}$ (line 2), and the component relation $C$ with the values of these placeholders, where the values of placeholders P.t.A for the attribute $A$ must satisfy the selection condition (line 3 ). If a placeholder P.t. $A$ has no value satisfying the selection condition, then $t$ is removed from $P^{0}$ (line 6) and all placeholders of $t$ are removed from $F$ (line 5) together with their values from $C$ (line 4).

Many of the standard query optimization techniques are also applicable in our context. For our experiments reported in Section 8, we performed the following optimizations on the sequences of SQL statements obtained as rewritings. For the evaluation of a query involving join, we merge the product and the selections with join conditions and distribute projections and selections to the operands. When evaluating a query involving several selections and projections on the same relation, we again merge these operators and perform the steps of the algorithm of Figure 16 only once. We further tuned the query evaluation by employing indices and materializing often used temporary results.

\section{Confidence Computation in Probabilistic WSDs}

Section 4 discussed algorithms for evaluating relational algebra queries on top of WSDs. Since we consider queries that transform worlds, the algorithms were independent of whether or not probabilities were stored with the data. A different class of queries are ones that compute confidence of tuples. The confidence of a tuple $t$ in the result of a query $Q$ is defined as the sum of the probabilities of the worlds that contain $t$ in the answer to $Q$. Clearly, iterating over all possible worlds is infeasible. We therefore adopt an approach where we only iterate over the local worlds of the relevant components.

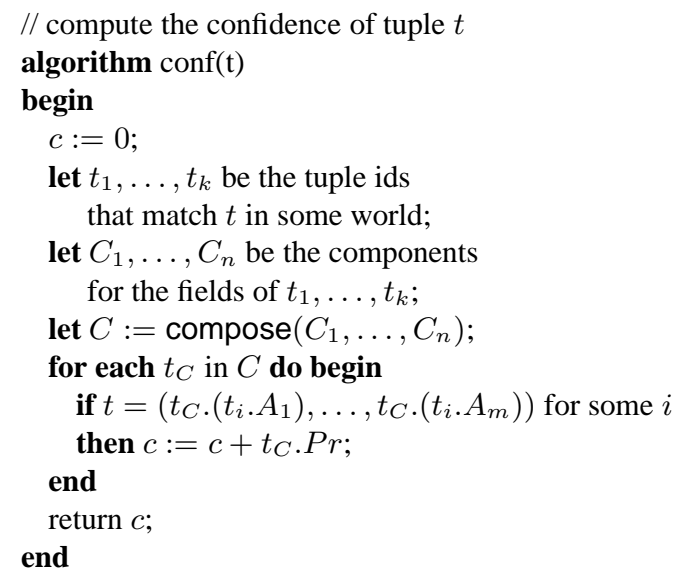

\section{Figure 17. Computing confidence of possible tuples.}

Figure 17 shows an algorithm for computing the confidence of tuple $t$ of schema $\left(A_{1}, \ldots, A_{m}\right)$. It first finds those tuple ids $t_{1}, \ldots, t_{k}$ that match the given tuple $t$ in some world and composes all components defining fields of those tuple ids into one component $C$. A world that contains $t$ is thus obtained whenever we select a local world from $C$ that makes the value of some tuple id $t_{i}, 1 \leq i \leq m$, equal to $t$. Fixing a local world in $C$ defines a set of possible worlds - the ones that share the values specified by the selected local world. The probability of this set of worlds is given in the $\operatorname{Pr}$ field of the local world. Since the local worlds of a component define non-overlapping sets of worlds, to compute the confidence of $t$ we need to sum up the probabilities of those local worlds that define $t$.

Note that the algorithms for computing tuple confidence in [12] rely heavily on the fact that input tuples are indepen- 
dent. Tuple confidence is computed during the evaluation of the query in question to avoid having to store intermediate results. This restricts the supported types of queries and the query plans that can be used. In probabilistic WSDs on the other hand, the query evaluation can be completely decoupled from confidence computation, since the latter form a strong representation system. For the same reason we need no independence assumptions about the input data.

The algorithm of Figure 17 does not explore possible independence between tuples. One can design a better approach in the following way. In a probabilistic WSD each component id corresponds to an independent random variable, whose possible outcomes are the local worlds of the component. We will call a world-set descriptor (wsdescriptor) a set

$$
\left\{\left(C_{1}, L_{1}\right), \ldots,\left(C_{n}, L_{n}\right)\right\}
$$

where $C_{i}$ is a component id, $L_{i}$ is a local world id of $C_{i}$, and no two elements $\left(C_{i}, L_{i}\right),\left(C_{j}, L_{j}\right)$ of the set exist with $C_{i}=C_{j}$ and $L_{i} \neq L_{j}$. A ws-descriptor defines, as its name suggests, a set of possible worlds, whose probability can be computed as the product of the probabilities of the selected local worlds:

$$
P\left(\left\{\left(C_{1}, L_{1}\right), \ldots,\left(C_{n}, L_{n}\right)\right\}\right)=\prod_{i=1}^{n} P\left(C_{i}, L_{i}\right)
$$

A ws-descriptor that specifies a local world for each component id of a probabilistic WSD corresponds to a single world. For computing tuple confidence we need to also consider sets of ws-descriptors. A ws-descriptor set defines a set of possible worlds - the union of the worlds defined by each descriptor in the set. Given a fixed tuple $t$ and a probabilistic world-set decomposition $\mathcal{W}$ representing the answer $R$ to query $Q$, we compute a ws-descriptor set $D$ for the worlds containing $t$ in the following way. Let $t_{i}$ be a tuple id of $R$ and $C_{i_{1}}, \ldots, C_{i_{j}}$ be the components of $\mathcal{W}$ that define fields of $R . t_{i}$. If the value of $t_{i}$ is $t$ when we fix the local world of $C_{i_{k}}$ to be $L_{i_{k}}$ for $1 \leq k \leq j$, respectively, then $D$ contains the ws-descriptor $\left\{\left(C_{i_{1}}, L_{i_{1}}\right), \ldots,\left(C_{i_{j}}, L_{i_{j}}\right)\right\}$. The confidence of $t$ is then computed as the probability of the worlds defined by $D$. Computing tuple confidence can be reduced to computing the probability of a formula in disjunctive normal form, which is known to have \#P complexity. This follows from the mutual reducibility of the problem of computing the probability of the union of the (possibly overlapping) world-sets represented by a set of wsdescriptors and of the \#P-complete problem of counting the number of satisfying assignments of Boolean formulas in disjunctive normal form. Indeed, we can encode a set of $k$ ws-descriptors $\left\{\left\{\left(C_{i_{1}}, L_{i_{1}}\right), \ldots,\left(C_{i_{j}}, L_{i_{j}}\right)\right\}\right\}, 1 \leq i \leq k$ as a formula $\bigvee_{1 \leq i \leq k}\left(C_{i_{1}}=L_{i_{1}} \wedge \ldots \wedge C_{i_{j}}=L_{i_{j}}\right)$. Different optimization techniques exist for computing the probability of a boolean formula, such as variable elimination and Monte Carlo approximations [19].

Remark 6.1 The U-relations of [5] associate each possible combination of values with a ws-descriptor. In WSDs and UWSDTs on the other hand a combination of values is associated with a single pair of component and local world id. Thus WSDs form a special case of U-relations with dependency vectors of size one.

We next consider the operator possible that computes the tuples appearing in at least one world of the world-set. Formally, if $R$ is a relation name and $\mathbf{A}$ - a world-set, the operator possible is defined as:

$$
\operatorname{possible}(R)(\mathbf{A}):=\left\{t \mid \mathcal{A} \in \mathbf{A}, t \in R^{\mathcal{A}}\right\}
$$

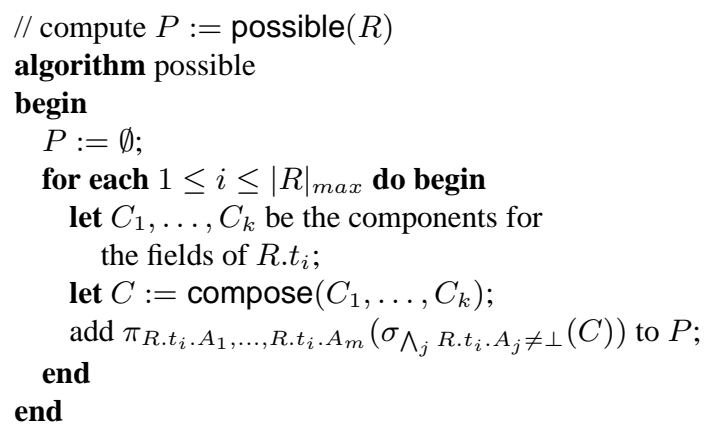

\section{Figure 18. Computing possible tuples.}

Figure 18 shows an algorithm for computing possible tuples in the non-probabilistic case. For each tuple id $t_{i}$ for $R$ we compose the components defining fields of $t_{i}$ to obtain the possible values for $t_{i}$.

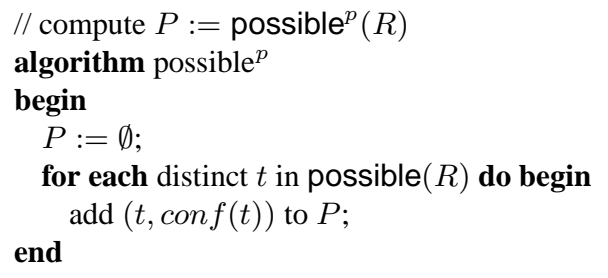

Figure 19. Computing possible tuples together with their confidence.

In the probabilistic case the operator possible can be extended to compute the confidence of the possible tuples. To 
do that, we compute the confidence of each tuple $t$, which is a possible answer to $Q$. Figure 19 shows an algorithm implementing the operator possible in the probabilistic case that computes the possible tuples together with their confidence. For computing the confidence $\operatorname{con} f(t)$ of tuple $t$ we can plug in any exact or approximate algorithm, e.g. the one from Figure 17

Example 6.2 Consider the probabilistic WSD of Figure 4. query $Q=\pi_{S}(R)$, and tuple $t=(185)$. Let $C_{1}$ denote the first component. This component represents the answer to the projection query. There are two tuple ids whose values match the given tuple $t$, and they are already defined in the same component $C_{1}$. To compute the confidence of $t$ we therefore need to sum up the probabilities of the first and second local world, obtaining $0.2+0.4=0.6$. The following table contains the possible tuples in the answer to $Q$ together with their confidence:

\begin{tabular}{c|c|c}
$Q$ & $\mathrm{~S}$ & conf \\
\hline & 185 & 0.6 \\
& 186 & 0.6 \\
& 785 & 0.8
\end{tabular}

\section{Normalizing probabilistic WSDs}

The normalization of a WSD is the process of finding an equivalent probabilistic WSD that takes the least space among all its equivalents. Examples of not normalized WSDs are non-maximal WSDs or WSDs defining invalid tuples (i.e., tuples that do not appear in any world). Note that removing invalid tuples and maximizing world-set decompositions can be performed in polynomial time [6].

Figure 20 gives three algorithms that address these normalization problems. The third algorithm scans for identical tuples in a component and compresses them into one by summing up their probabilities.

Example 7.1 The WSD of Figure 11 (a) has only $\perp$-values for P.t. $t_{2}$.C. This means that the tuple $t_{2}$ of $P$ is absent (or invalid) in all worlds and can be removed. The equivalent WSD of Figure 21 shows the result of this operation. Similar simplifications apply to the WSD of Figure 11(b), where tuples $t_{2}$ and $t_{3}$ are invalid.

Example 7.2 The 4-WSD of Figure 13 admits the equivalent 5-WSD, where the third component is decomposed into two components. This non-maximality case cannot appear for UWSDTs, because all but the first component contain only one tuple and are stored in the template relation, where no component merging occurs.

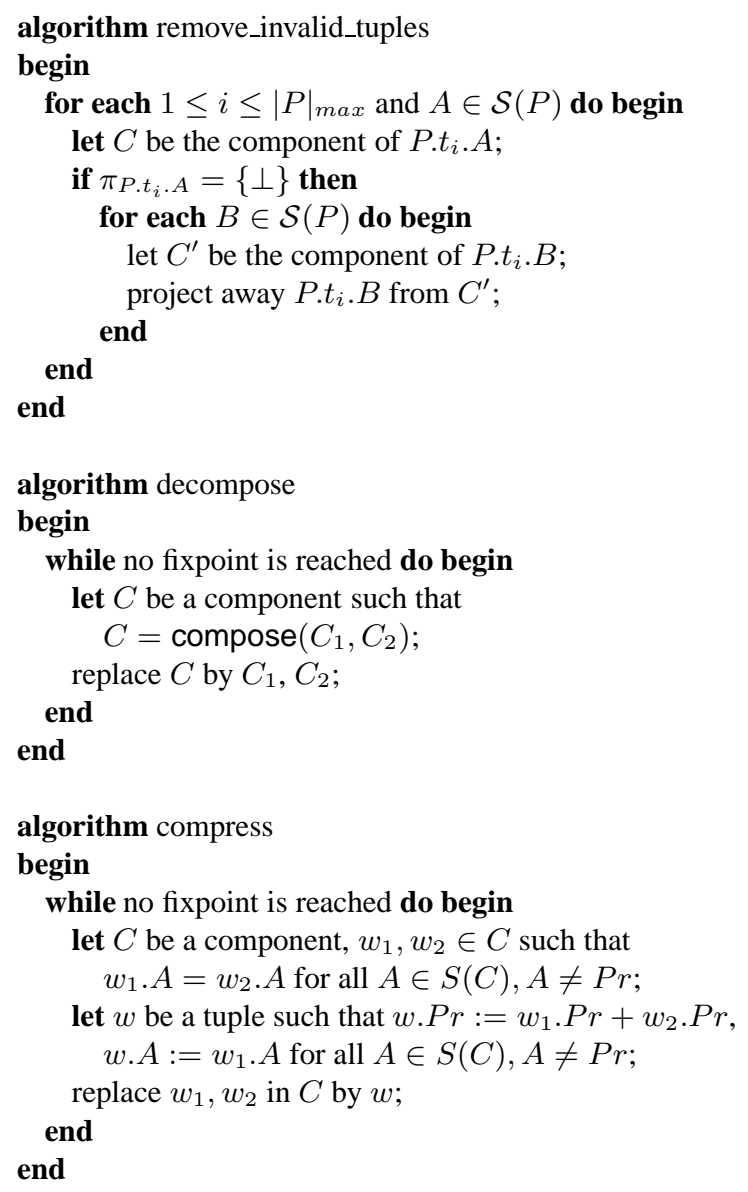

Figure 20. Algorithms for WSD normalization.

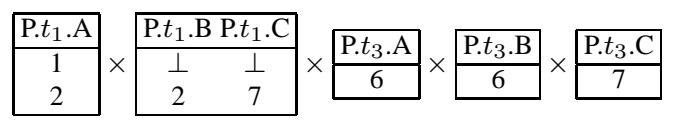

Figure 21. Normalization of WSD of Figure 11(a).

\section{Experimental Evaluation}

The literature knows a number of approaches to representing incomplete information databases, but little work has been done so far on expressive yet efficient representation systems. An ideal representation system would allow a large set of possible worlds to be managed using only a small overhead in storage space and query processing time when compared to a single world represented in a conventional way. In the previous sections we presented the first step towards this goal. This section reports on experiments 
with a large census database with noise represented as a UWSDT.

Setting. The experiments were conducted on a $3 \mathrm{GHz} /$

2GB Pentium machine running Linux 2.6.8 and PostgreSQL 8.0.

Datasets. The IPUMS 5\% census data (Integrated Public Use Microdata Series, 1990) [20] used for the experiments is the publicly available 5\% extract from the 1990 US census, consisting of 50 (exclusively) multiple-choice questions. It is a relation with 50 attributes and 12491667 tuples (approx. 12.5 million). The size of this relation stored in PostgreSQL is ca. 3 GB. We also used excerpts representing the first $0.1,0.5,1,5,7.5$, and 10 million tuples.

Adding Incompleteness. We added incompleteness as follows. First, we generated a large set of possible worlds by introducing noise. After that, we cleaned the data by removing worlds inconsistent with respect to a given set of dependencies. Both steps are detailed next.

We introduced noise by replacing some values with orset:2. We experimented with different noise densities: $0.005 \%, 0.01 \%, 0.05 \%, 0.1 \%$. For example, in the $0.1 \%$ scenario one in 1000 fields is replaced by an or-set. The size of each or-set was randomly chosen in the range $[2, \min (8, \operatorname{size})]$, where size is the size of the domain of the respective attribute (with a measured average of $3.5 \mathrm{val}-$ ues per or-set). In one scenario we had far more than $2^{624449}$ worlds, where 624449 is the number of the introduced orsets and 2 is the minimal size of each or-set (cf. Figure 22).

We then performed data cleaning using 12 equality generating dependencies, representing real-life constraints on the census data. Note that or-set relations are not expressive enough to represent the cleaned data with dependencies.

To remove inconsistent worlds with respect to given dependencies, we adapted the Chase technique [2] to the context of UWSDTs. We explain the Chase by an example. Consider the dependency WWII $=1 \Rightarrow$ MILITARY $!=4$ that requires people who participated in the second world war to have completed their military service. Assume now the dependency does not hold for a tuple $t$ in some world and let $C_{1}$ and $C_{2}$ be the components defining $t$.WWII and $t$.MILITARY, respectively. First, the Chase computes a component $C$ that defines both $t$.WWII and $t$.MILITARY. In case $C_{1}$ and $C_{2}$ are different, they are replaced by a new component $C=C_{1} \times C_{2}$; otherwise, $C$ is $C_{1}$. The Chase removes then from $C$ all inconsistent worlds $w$, i.e., worlds where $w$.WWII $=1$ and $w$.MILITARY $=4$. Repeating these steps iteratively for each dependency on a given UWSDT yields a UWSDT satisfying all dependencies.

Figure 22 shows the effect of chasing our dependencies on the 12.5 million tuples and varying placeholder density. As a result of merging components, the number of com-

\footnotetext{
${ }^{2}$ We consider it infeasible both to iterate over all worlds in secondary storage, or to compute UWSDT decompositions by comparing the worlds.
}

\begin{tabular}{|c|c|c|c|c|c|}
\hline & Density & $0.005 \%$ & $0.01 \%$ & $0.05 \%$ & $0.1 \%$ \\
\hline Initial & \#comp & 31117 & 62331 & 312730 & 624449 \\
\hline \multirow{4}{*}{$\begin{array}{l}\text { After } \\
\text { chase }\end{array}$} & \#comp & 30918 & 61791 & 309778 & 612956 \\
\hline & $\#$ comp $>1$ & 249 & 522 & 2843 & 10880 \\
\hline & $|C|$ & 108276 & 217013 & 1089359 & 2150935 \\
\hline & $|R|$ & $12.5 \mathrm{M}$ & $12.5 \mathrm{M}$ & $12.5 \mathrm{M}$ & $12.5 \mathrm{M}$ \\
\hline After & \#comp & 702 & 1354 & 7368 & 14244 \\
\hline \multirow[t]{3}{*}{$Q_{1}$} & $\#$ comp $>1$ & 1 & 4 & 40 & 158 \\
\hline & $|C|$ & 1742 & 3625 & 19773 & 37870 \\
\hline & $|R|$ & 46600 & 46794 & 48465 & 50499 \\
\hline After & \#comp & 25 & 56 & 312 & 466 \\
\hline \multirow[t]{3}{*}{$Q_{2}$} & $\#$ comp $>1$ & 0 & 1 & 8 & 9 \\
\hline & $|C|$ & 93 & 269 & 1682 & 2277 \\
\hline & $|R|$ & 82995 & 83052 & 83357 & 83610 \\
\hline After & \#comp & 38 & 76 & 370 & 742 \\
\hline \multirow[t]{3}{*}{$Q_{3}$} & $\#$ comp $>1$ & 0 & 0 & 0 & 0 \\
\hline & $|C|$ & 89 & 202 & 1001 & 2009 \\
\hline & $|R|$ & 17912 & 17936 & 18161 & 18458 \\
\hline After & \#comp & 1574 & 3034 & 15776 & 30729 \\
\hline \multirow[t]{3}{*}{$Q_{4}$} & $\#$ comp $>1$ & 11 & 28 & 127 & 557 \\
\hline & $|C|$ & 4689 & 9292 & 48183 & 94409 \\
\hline & $|R|$ & 402345 & 402524 & 404043 & 405869 \\
\hline After & \#comp & 3 & 10 & 53 & 93 \\
\hline \multirow[t]{3}{*}{$Q_{5}$} & $\#$ comp $>1$ & 3 & 10 & 53 & 93 \\
\hline & $|C|$ & 1221 & 5263 & 33138 & 50780 \\
\hline & $|R|$ & 150604 & 173094 & 274116 & 393396 \\
\hline After & \#comp & 97 & 189 & 900 & 1888 \\
\hline \multirow[t]{3}{*}{$Q_{6}$} & $\#$ comp $>1$ & 0 & 0 & 0 & 0 \\
\hline & $|C|$ & 516 & 1041 & 4993 & 10182 \\
\hline & $|R|$ & 229534 & 230113 & 234335 & 239488 \\
\hline
\end{tabular}

\section{Figure 22. UWSDTs characteristics for $12.5 \mathrm{M}$ tuples.}

ponents with more than one placeholder (\#comp $>1$ ) grows linearly with the increase of placeholder density, reaching about $1.7 \%$ of the total number of components (\#comp) in the $0.1 \%$ case. A linear increase is witnessed also by the chasing time when the number of tuples is also varied.

Queries. Six queries were chosen to show the behavior of relational operators combinations under varying selectivities (cf. Figure 23). Query $Q_{1}$ returns the entries of US citizens with $\mathrm{PhD}$ degree. The less selective query $Q_{2}$ returns the place of birth of US citizens born outside the US that do not speak English well. Query $Q_{3}$ retrieves the entries of widows that have more than three children and live in the state where they were born. The very unselective query $Q_{4}$ returns all married persons having no children. Query $Q_{5}$ uses query $Q_{2}$ and $Q_{3}$ to find all possible couples of widows with many children and foreigners with limited English language proficiency in US states with IPUMS index greater than 50 (i.e., eight 'states', e.g., Washington, Wisconsin, Abroad). Finally, query $Q_{6}$ retrieves the places of birth and work of persons speaking English well.

Figure 22 describes some characteristics of the answers to these queries when applied on the cleaned $12.5 \mathrm{M}$ tuples of IPUMS data: the total number of components 


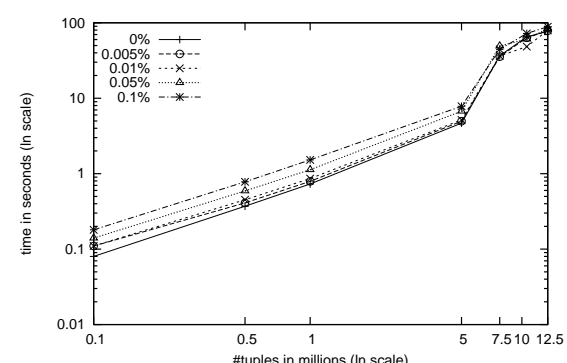

(a) Query $Q_{1}$

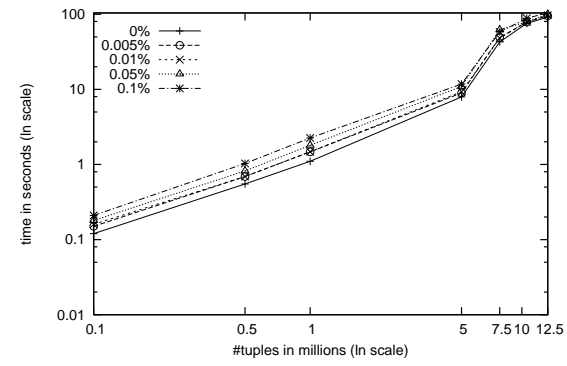

(d) Query $Q_{4}$

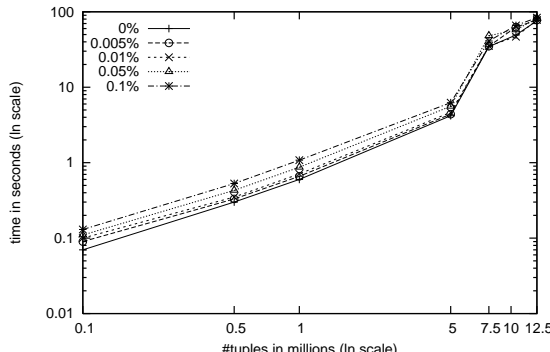

(b) Query $Q_{2}$

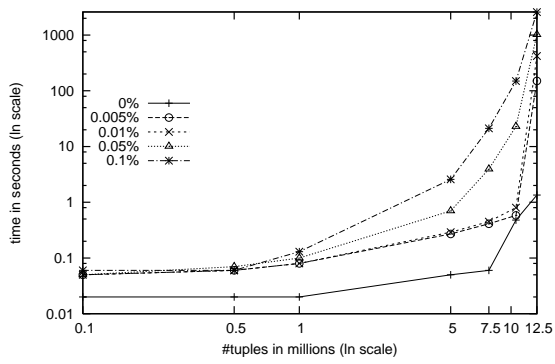

(e) Query $Q_{5}$

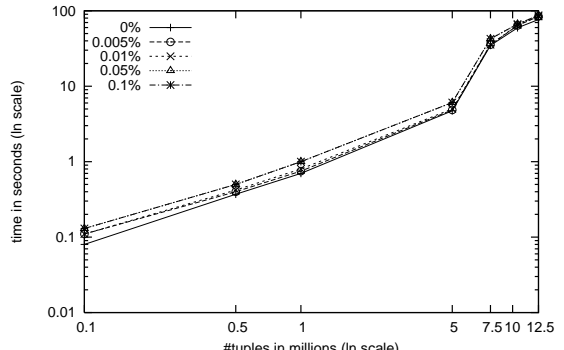

(c) Query $Q_{3}$

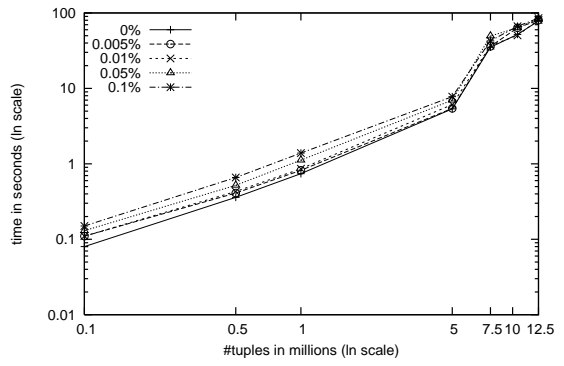

(f) Query $Q_{6}$

Figure 24. The evaluation time for queries of Figure 23 on UWSDTs of various sizes and densities.

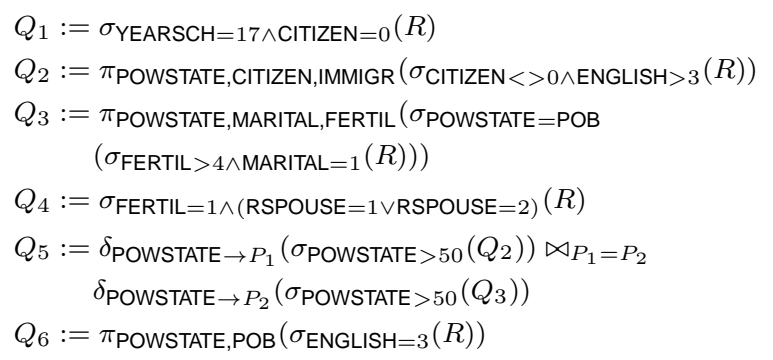

Figure 23. Queries on IPUMS census data.

(\#comp) and of components with more than one placeholder (\#comp >1), the size of the component relation $C$, and the size of the template relation $R$. One can observe that the number of components increases linearly with the placeholder density and that compared to chasing, query evaluation leads to a much smaller amount of component merging.

Figure 24 shows that all six queries admit efficient and scalable evaluation on UWSDTs of different sizes and placeholder densities. For accuracy, each query was run ten times, and the median time for computing and storing the answer is reported. The evaluation time for all queries but $Q_{5}$ on UWSDTs follows very closely the evaluation time in the one-world case. The one-world case corresponds to density $0 \%$ in our diagrams, i.e., when no placeholders are created in the template relation and consequently there are no components. In this case, the original queries (that is, not the rewritten ones) of Figure 23 were evaluated only on the (complete) template relation.

An interesting issue is that all diagrams of Figure 24 show a substantial increase in the query evaluation time for the $7.5 \mathrm{M}$ case. As the jump appears also in the oneworld case, it suggests poor memory management of Postgres in the case of large tables. We verified this statement by splitting the $12.5 \mathrm{M}$ table into chunks smaller than $5 \mathrm{M}$ and running query $Q_{1}$ on those chunks to get partial answers. The final answer is represented then by the union of each UWSDT relation from these partial answers.

Although the evaluation of join conditions on UWSDTs can require theoretically exponential time (due to the composition of some components), our experiments suggest that they behave well in practical cases, as illustrated in Figures 24 (c) and (e) for queries $Q_{3}$ and $Q_{5}$ respectively. Note that the time reported for $Q_{5}$ does not include the time to evaluate its subqueries $Q_{2}$ and $Q_{3}$.

In summary, our experiments show that UWSDTs behave very well in practice. We found that the size of UWSDTs obtained as query answers remains close to that of one of their worlds. Furthermore, the processing time for queries on UWSDTs is comparable to processing one world. The explanation for this is that in practice there are rather few differences between the worlds. This keeps the mapping and component relations relatively small and the lion's share of the processing time is taken by the templates, whose sizes are about the same as of a single world. 


\section{Application Scenarios}

Our approach is designed to cope with large sets of possible worlds, which exhibit local dependencies and large commonalities. This data pattern can be found in many applications. In addition to the census scenario used in Section 8, we next discuss two further application scenarios that can profit from our approach. As for the census scenario, we consider it infeasible both to iterate over all possible worlds in secondary storage, or to compute UWSDT decompositions by comparing the worlds. Thus we also outline how our UWSDTs can be efficiently computed.

Inconsistent databases. A database is inconsistent if it does not satisfy given integrity constraints. Sometimes, enforcing the constraints is undesirable. One approach to manage such inconsistency is to consider so-called minimal repairs, i.e., consistent instances of the database obtained with a minimal number of changes [7]. A repair can therefore be viewed as a possible (consistent) world. The number of possible minimal repairs of an inconsistent database may in general be exponential; however, they substantially overlap. For that reason repairs can be easily modeled with UWSDTs, where the consistent part of the database is stored in template relations and the differences between the repairs in components. Current work on inconsistent databases [7] focuses on finding consistent query answers, i.e., answers appearing in all possible repairs (worlds). With our approach we can provide more than that, as the answer to a query represents a set of possible worlds. In this way, we preserve more information that can be further processed using querying or data cleaning techniques.

Medical data. Another application scenario is modeling information on medications, diseases, symptoms, and medical procedures, see, e.g., [1]. A particular characteristic of such data is that it contains a big number of clusters of interdependent data. For example, some medications can interact negatively and are not approved for patients with some diseases. Particular medical procedures can be prescribed for some diseases, while they are forbidden for others. In the large set of possible worlds created by the complex interaction of medications, diseases, procedures, and symptoms, a particular patient record can represent one or a few possible worlds. Our approach can keep interdependent data within components and independent data in separate components. One can ask then for possible patient diagnostics, given an incompletely specified medical history of the patient, or for commonly used medication for a given set of diseases.

In [1] interdependencies of medical data are modeled as links. A straightforward and efficient approach to wrap such data in UWSDTs is to follow the links and create one component for all interrelated values. Additionally, each different kind of information, like medications, diseases, is stored in a separate template relation.

\section{References}

\section{[1] http://www.medicinenet.com.}

[2] Serge Abiteboul, Richard Hull, and Victor Vianu. Foundations of Databases. Addison-Wesley, 1995.

[3] Serge Abiteboul, Paris Kanellakis, and Gösta Grahne. On the representation and querying of sets of possible worlds. Theor. Comput. Sci., 78(1):158-187, 1991.

[4] Periklis Andritsos, Ariel Fuxman, and Renee J. Miller. Clean answers over dirty databases: A probabilistic approach. In Proc. ICDE, 2006.

[5] Lyublena Antova, Thomas Jansen, Christoph Koch, and Dan Olteanu. Fast and simple relational processing of uncertain data. In Proc. ICDE, 2008. to appear.

[6] Lyublena Antova, Christoph Koch, and Dan Olteanu. World-set decompositions: Expressiveness and efficient algorithms. In Proc. ICDT, 2007.

[7] Marcelo Arenas, Leopoldo E. Bertossi, and Jan Chomicki. Consistent query answers in inconsistent databases. In Proc. PODS, pages 68-79, 1999.

[8] Omar Benjelloun, Anish Das Sarma, Alon Halevy, and Jennifer Widom. ULDBs: Databases with uncertainty and lineage. In Proc. VLDB, 2006.

[9] Philip Bohannon, Wenfei Fan, Michael Flaster, and Rajeev Rastogi. "A Cost-Based Model and Effective Heuristic for Repairing Constraints by Value Modification. In Proc. SIGMOD, 2005.

[10] Andrea Calí, Domenico Lembo, and Riccardo Rosati. On the decidability and complexity of query answering over inconsistent and incomplete databases. In Proc. PODS, pages 260-271, 2003.

[11] Reynold Cheng, Sarvjeet Singh, and Sunil Prabhakar. U-DBMS: A database system for managing constantly-evolving data. In Proc. VLDB, pages 12711274, 2005.

[12] Nilesh Dalvi and Dan Suciu. Efficient query evaluation on probabilistic databases. In Proc. VLDB, pages 864-875, 2004.

[13] H. Galhardas, D. Florescu, D. Shasha, and E Simon. "AJAX: An Extensible Data Cleaning Tool". In Proc. SIGMOD, 2000.

[14] Rahul Gupta and Sunita Sarawagi. Creating probabilistic databases from information extraction models. In Proc. VLDB, 2006. 
[15] T. Imielinski and W. Lipski. Incomplete information in relational databases. Journal of ACM, 31:761-791, 1984.

[16] T. Imielinski, S. Naqvi, and K. Vadaparty. Incomplete objects - a data model for design and planning applications. In Proc. SIGMOD, pages 288-297, 1991.

[17] Erhard Rahm and Hong Hai Do. "Data Cleaning: Problems and Current Approaches". IEEE Data Engineering Bulletin, 2000.

[18] V. Raman and J.M. Hellerstein. "Potter's Wheel: An Interactive Data Cleaning System". In Proc. VLDB, 2001.

[19] Christopher Re, Nilesh Dalvi, and Dan Suciu. Efficient Top-k Query Evaluation on Probabilistic Data. In Proc. ICDE, 2007.

[20] Steven Ruggles, Matthew Sobek, Trent Alexander, Catherine A. Fitch, Ronald Goeken, Patricia Kelly Hall, Miriam King, and Chad Ronnander. Integrated public use microdata series: V3.0, 2004. http://www.ipums.org.

[21] Anish Das Sarma, Omar Benjelloun, Alon Halevy, and Jennifer Widom. Working models for uncertain data. In Proc. ICDE, 2006. 